\title{
Análise da potência absoluta na banda gama no córtex pré-frontal após diagonal de membro superior
}

\author{
Analysis of absolute power in the gamma band in the \\ prefrontal cortex after upper limb diagonal
}

Alice Aragão de Araújo ${ }^{1}$, Janaína de Moraes Silva ${ }^{2}$, Tassiane Maria Alves Pereira ${ }^{3}$, Rayele Moreira ${ }^{4}$, Monara Nunes ${ }^{4}$, Kaline Rocha ${ }^{4}$, Silmar Silva Teixeira ${ }^{5}$, Marco Orsini ${ }^{6}$, Adalgiza Mafra Moreno7, Aluana Santana Carlos ${ }^{7}$; Pedro Ribeiro ${ }^{8}$, Bruna Velasques ${ }^{8}$, Victor Hugo Bastos ${ }^{9}$; Eduardo Jorge Custódio da Silva ${ }^{10}$, Ulisses Cerqueira Linhares ${ }^{11}$, Rossano Fiorelli ${ }^{11}$, Maria Izabel Muller ${ }^{11}$, Giseli Roque de Souza ${ }^{11}$

1.Fisioterapeuta, Universidade Federal do Piauí (UFPI), Parnaíba-PI, Brasil.

2.Docente, Laboratório de Mapeamento e Plasticidade Cerebral, Laboratório de Mapeamento Cerebral e Funcionalidade, UFPI-CMRV-LAMPLACE, Universidade Estadual do Piauí (UESPI), Teresina-PI, Brasil.

3. Faculdade Maurício de Nassau (UNINASSAU), Teresina-Piauí, Brasil.

4.Fisioterapeuta, Laboratório de Mapeamento e Plasticidade Cerebral, Laboratório de Mapeamento Cerebral e Funcionalidade, UFPI-CMRV-LAMPLACE, Universidade Estadual do Piauí (UESPI), Teresina-PI, Brasil.

5.Laboratório de Mapeamento e Plasticidade Cerebral - UFPI-CMRV-LAMPLACE, Parnaíba-PI, Brasil; 6. Fisioterapeuta, Laboratório de Mapeamento e Plasticidade Cerebral, Laboratório de Mapeamento Cerebral e Funcionalidade, UFPI-CMRV-LAMPLACE, Universidade Estadual do Piauí (UESPI), Teresina-PI, Brasil. Universidade de Vassouras - Mestrado em Ciências Aplicadas em Saúde. Universidade Iguaçu - RJ 7. Universidade Iguaçu - UNIG - Rio de Janeiro.

8. Docente da Universidade Federal do Rio de Janeiro (UFRJ), Rio de Janeiro-RJ, Brasil.

8.Laboratório de mapeamento cerebral e integração sensório-motora - IPUB/UFRJ, Rio de Janeiro-RJ, Brasil.

9.Laboratório de Mapeamento Cerebral e Funcionalidade - UFPI-CMRV-LAMCEF, Parnaíba-PI, Brasil;

10. Hospital Pedro Ernesto - UERJ - Departamento de Neurolofisiologia, Brasil.

11. Universidade de Vassouras - Rio de Janeiro - Brasil.

\section{Resumo}

No âmbito da fisioterapia, movimentos realizados em diagonal por meio de padrões específicos visam a recuperação funcional dos pacientes. Esses exercícios terapêuticos são utilizados com o intuito de evitar lesões, sendo benéficos nos programas que promovam alongamento e fortalecimento. No entanto, embora movimentos em diagonal sejam bastante utilizados na prática clínica, as repercussões corticais provocadas por esse tipo de movimento ainda são desconhecidas. Objetivo. O objetivo deste estudo foi investigar quais as repercussões eletrofisiológicas dos movimentos em diagonal por meio da análise da potência absoluta na banda gama no córtex pré-frontal. Método. A amostra foi composta por 20 voluntárias, com idade entre 18 a 28 anos, destras, não praticantes de atividade física e com Índice de Massa Corpórea (IMC) entre 18,50 a 24,99. Foram utilizadas ANOVA mista de um, dois e três fatores seguido do teste de post hoc. Resultados. Observamos interação entre os fatores grupos, áreas corticais e momentos. Conclusão. Concluímos que o treinamento em diagonal promove alterações na atividade cortical uma vez que um aumento da potência absoluta na banda gama no córtex pré-frontal, à medida que o sujeito realizava a tarefa, foi observado.

Unitermos. EEG; Banda Beta; Potência Absoluta

\section{Abstract}

Within the physical therapy, movements performed in the diagonal position by specific patterns are used for the functional recovery of patients. These therapeutic 
exercises are used in order to avoid injury, being beneficial in programs which promote stretching and strengthening. However, although diagonal movements are widely used in clinical practice, the cortical effects caused by this type of movement, in particular, are still unknown. Objective. The objective of this study was to investigate what are the electrophysiological effects of movements in diagonal through the analysis of absolute power in the gamma band in the prefrontal cortex. Method. This study had a sample of 20 female volunteers at the age of 18 to 28 years old, right-handed, who do not practice physical activity, and with Body Mass Index (BMI) from 18.50 to 24.99. There were used Analysis of variance (ANOVA) mixed of one-way, two-way or three-way factors followed by the post hoc test. Results. We noticed an interaction between the group factors, the cortical areas and the moments. Conclusion. We conclude that the diagonal training promotes changes in cortical activity since an increase of the absolute power in the range band in prefrontal cortex, as the subject performed the task was noticed.

Keywords. EEG; Gamma Band; Absolute power

Trabalho realizado Universidade Federal do Piauí (UFPI), Parnaíba-PI, Brasil.

Conflito de interesse: não Recebido em: 2018

Aceito em: 2018

Endereço para correspondência: Janaína M Silva. R. Olavo Bilac, 2335. Centro (Sul). Teresina-PI, Brasil. CEP 64001-280. E-mail: fisiojanainams@gmail.com

\section{INTRODUÇÃO}

Movimentos realizados em diagonal são utilizados no âmbito da fisioterapia, para a recuperação funcional dos pacientes e são aplicados principalmente nos programas que visam alongamento e fortalecimento ${ }^{1-4}$. O conceito de tratamento utilizando diagonais destaca que todos os indivíduos, incluindo aqueles com alterações físicas têm um potencial funcional ${ }^{5,6}$ ao envolver a estimulação dos proprioceptores musculares e articulares ${ }^{6-11}$. Neste contexto, a tarefa motora promove modulações da atividade cortical, no entanto, embora movimentos em diagonal sejam bastante utilizados na prática clínica e sua repercussão mioelétrica seja elucidada na literatura, as repercussões corticais provocadas por esse tipo de movimento, ainda são desconhecidas ${ }^{12}$. 
A realização de uma tarefa motora envolve a intenção de realizá-la, a preparação, planejamento e execução real do movimento. Toda essa organização promove modificações nas oscilações corticais que podem ser analisadas com a eletroencefalografia $(E E G)^{13}$. Esta ferramenta é frequentemente usada para o diagnóstico e acompanhamento clínico de demência, epilepsia, distúrbios do sono e outras condições clínicas ${ }^{14-17}$. No entanto, no âmbito da fisioterapia, tem sido empregada para avaliar a atividade cortical a fim de compreender o comportamento cortical durante tarefas motoras e cognitivas relacionadas com a reabilitação ${ }^{18-22}$.

O emprego dos padrões de movimento em diagonal na reabilitação tem sido aplicado com expressivos resultados para retorno da atividade motora e melhorar a sensibilidade após lesão neurológica sucesso. Desse modo, o entendimento que este estudo fornece pode implicar em conhecimentos que otimizem a utilização do tratamento com diagonais de membro superior nas disfunções musculoesqueléticas. Em especial, analisamos a banda gama (40 a $100 \mathrm{~Hz}$ ) do $\mathrm{EEG}^{23}$, a qual está relacionada com processo, a memória e integração espacial/temporal ${ }^{22,24-27}$. Diante disso, o objetivo deste estudo foi investigar quais são as repercussões eletrofisiológicas dos movimentos em diagonal por meio da análise da potência absoluta na banda gama no córtex pré-frontal. 


\section{MÉTODO}

Estudo do tipo transversal controlado, conduzido no Laboratório de Mapeamento Cerebral e Funcionalidade (LAMCEF) da Universidade Federal do Piauí, Campus de Parnaíba, Piauí, Brasil. A amostra contou com 20 voluntárias, com idade entre 18 a 28 anos, destras ${ }^{28}$, não praticantes de atividade física ${ }^{29}$ e com Índice de Massa Corpórea (IMC) entre 18,50 a 24,99; considerado dentro da faixa de normalidade ${ }^{30}$. A fim de garantir maior homogeneidade da amostra foram selecionados apenas estudantes do sexo feminino, e que não estavam familiarizadas com o princípio básico da Facilitação Neuromuscular Proprioceptiva (FNP), que são prioritariamente realizados em diagonal.

Os critérios de exclusão foram doenças musculoesqueléticas ou articulares no membro superior direito, presença de quaisquer patologias cardíacas, pulmonares ou neurológicas. Indivíduos com limitação funcional para realizar movimentos resistidos, amputados e portadores de déficit sensorial ou cognitivo que limitasse a realização do movimento solicitado. No tocante ao EEG, as participantes não poderiam ter feito uso de drogas psicotrópicas ou psicoativas e sono inferior ao período de 6hrs na noite anterior ao experimento. As voluntárias que estavam dentro dos critérios de inclusão e concordaram em participar do experimento foram esclarecidas quanto ao procedimento experimental e confidencialidade da pesquisa e assinaram o Termo de Consentimento Livre e Esclarecido 
(TCLE). O estudo atende às determinações previstas para a pesquisa envolvendo humanos constantes na Declaração de Helsinque e na Resolução do Conselho Nacional de Saúde no 466/2012 com parecer consubstanciado do Comitê de Ética e Pesquisa (CEP), número 683.331.

\section{Eletroencefalografia quantitativa}

O sinal eletroencefalográfico foi captado com o aparelho BrainNet BNT 36 - EEG (EMSA-Instrumentos Médicos, Brasil). Uma touca elástica, tamanho adulto médio $(58-54 \mathrm{~cm})$ com eletrodos dispostos segundo o sistema internacional 10-20 e uma cinta elástica para fixação, foi utilizada para a captação, incluindo os eletrodos de referência posicionados nos lóbulos das orelhas (biauricular). A sala de captação estava isolada acústica e eletricamente (aterramento adequado) e a impedância da interface pele-eletrodo foi mantida entre 5 e $10 \mathrm{k} \Omega$. Os dados adquiridos tinham amplitude total menor que $100 \mu \mathrm{V}$. O sinal EEG foi filtrado analogicamente entre $0,1 \mathrm{~Hz}$ (passa-altas) e $100 \mathrm{~Hz}$ (passa-baixas), sendo digitalizado a $400 \mathrm{~Hz}$. Com o software de aquisição e controle (desenvolvido em Delphi 5.0), os dados brutos foram ainda filtrados digitalmente: notch $(60 \mathrm{~Hz})$, passaaltas de $0,3 \mathrm{~Hz}$ e passa-baixas de $30 \mathrm{~Hz}$. Os dados foram analisados no software Matlab e no programa EEGlab que consiste em uma ferramenta interativa para 0 processamento contínuo de eventos relacionados à EEG e 
outros dados eletrofisiológicos, incluindo análise de componentes independentes (ICA) ${ }^{31}$.

\section{Processamento dos dados}

Uma inspeção visual e análise de componentes independentes (ACI) foram aplicadas para identificar e remover todos os artefatos remanescentes, ou seja, piscada de olhos e movimentos oculares produzidos pela tarefa ${ }^{31,32}$. Os dados de eletrodos individuais que exibiram perda de contato com o escalpo ou alta impedância (>10K $\Omega$ ) não foram considerados. Um estimador clássico foi aplicado para a densidade de potência espectral (DPE), estimada a partir da Transformada de Fourier, que foi realizada pelo MATLAB R2009b (Matwords, Inc.).

\section{Procedimento experimental}

As participantes foram randomizadas em dois grupos: controle (G1), grupo diagonal de flexão, abdução e rotação externa com extensão de cotovelo, punho e dedos (G2). O movimento partiu de uma flexão de punho e dedos com a mão apoiada na perna contralateral. A tarefa foi executada em nove blocos com nove trilhas e realizada apenas com o membro dominante. Para identificar a dominância da lateralidade manual foi utilizado o inventário de Edimburg ${ }^{28}$. Os pés dos sujeitos ficaram apoiados no chão e o quadril em flexão de $90^{\circ}$.

As voluntárias receberam instruções prévias de uma avaliadora e foram treinadas para a correta realização da 
manobra. De início, o movimento foi demostrado pela avaliadora, em seguida, foi realizado passivamente (voluntária com o auxílio da avaliadora) e finalmente a voluntária executou o movimento ativamente. Ao perceber que a voluntária compreendeu e executou o movimento corretamente, deu-se início ao procedimento principal.

Ao receber um estímulo visual em um monitor posicionado a $60 \mathrm{~cm}^{33}$, as voluntárias executaram a tarefa solicitada, repetindo-a 81 vezes, divididas em nove blocos, com intervalo de 3 minutos de repouso entre eles. Durante este intervalo foram realizadas as captações eletroencefalográficas (Figura 1).

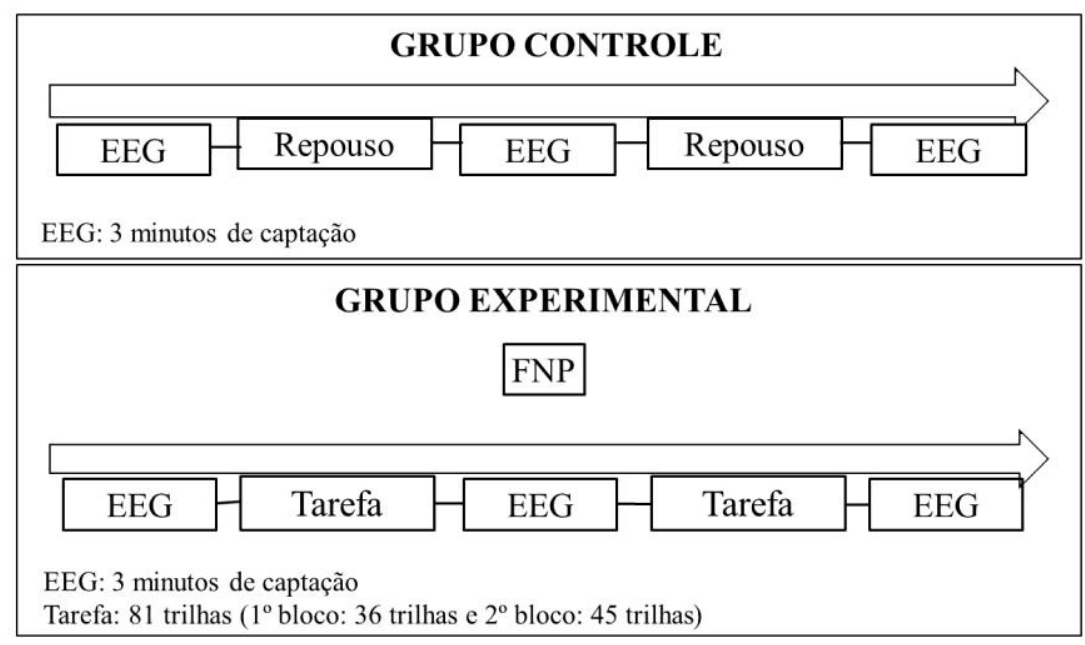

Figura 1. Desenho de Estudo do Procedimento

\section{Análise estatística}

O delineamento estatístico permitiu a análise da potência absoluta da banda gama antes, durante e após a 
realização das tarefas. As derivações selecionadas foram as relacionadas com o córtex pré-frontal dorsolateral esquerdo, linha média central e córtex pré-frontal dorsolateral direito (F3, FZ e F4). Assim, uma Three-way mixed factorial ANOVA foi realizada para a banda gama com fator intergrupos [Controle, FNP] e com fator intragrupos para área cortical (F3, FZ e F4) e momentos (antes, durante e após a tarefa).

Para avaliar a Three-way mixed factorial ANOVA usamos os critérios do teste de Mauchley's que avaliou a hipótese de esfericidade, e o procedimento de GreenhouseGeisser $(G-G \varepsilon)$ que corrigiu os graus de liberdade. A normalidade e homocedasticidade dos dados foram previamente verificadas pelos testes de Levene e ShapiroWilk $(p>0,05)$. As interações entre três fatores foram investigadas utilizando uma two-way ANOVA de medidas repedidas e uma one-way ANOVA de medidas repetidas seguido do teste de post hoc realizado com correção de Bonferroni. O tamanho do efeito também foi estimado como Eta parcial ao quadrado $\left(\eta^{2} \mathrm{p}\right)$. A potência estatística e o intervalo de confiança de 95\% (IC 95\%) foram calculados para as variáveis dependentes. Entretanto, quando uma one-way de medidas repetidas foi analisada separadamente para os fatores tempo e momento, foi considerada a significância estatística com um nível alfaBonferroni ajustado com $p=0,025$. A magnitude do efeito foi interpretada utilizando as recomendações sugeridas por Hopkins et al. (2009) ${ }^{34}$ : $0.0=$ trivial; 0.2 =pequeno; 
$0.6=$ moderado $; \quad 1.2=$ grande $; \quad 2.0=$ muito $\quad$ grande; $4.0=$ quase perfeita. A probabilidade de $5 \%$ para o erro tipo I foi adotada em todas as análises $(p \leq 0,05)$. Desse modo, para detectar se houve uma diferença real na população, 0 poder estatístico foi interpretado em 0,8 a $0,9=$ alto poder $^{35}$. As análises foram conduzidas utilizando o software SPSS para Windows versão 20.0 (SPSS Inc., Chicago, II, EUA).

\section{RESULTADOS}

Os resultados mostraram interação entre os fatores grupo, área cortical e momento $[F(4,2936)=112,143$; $\mathrm{p}<0,001 ; \eta^{2}=0,133$ e poder=1,00]. Ao analisá-la verificouse interação entre os fatores área cortical e momento tanto para o grupo controle $\left[F(4,1468)=101,292 ; p<0,001 ; \eta^{2}=\right.$ $0,216$ e poder $=1,00]$, quanto para o grupo FNP $\left[F(4,1468)=110,981 ; \quad p<0,001 ; \quad \eta^{2} p=0,232 ; \quad\right.$ poder $=$ $1,000]$.

A análise da interação demonstrou diferença significante para o fator área (Figura 2) no grupo controle, antes $\left[F(2,734)=273,856 ; p<0,001 ; \eta^{2} p=0,427 ;\right.$ poder $=$ $1,000]$, durante $\left[F(2,734)=275,125 ; p<0,001 ; \eta^{2} p=0,428\right.$; poder $=1,000]$ e depois da tarefa $[F(2,734)=366,968$; $p<0,001 ; \eta^{2} p=0,500 ;$ poder $\left.=1,000\right]$. O teste de post-hoc mostrou que antes da realização da tarefa a derivação F3 teve uma potência maior em 0,002 $\mu \mathrm{V}$ [(IC 95\%, 0,001 a $0,002), p<0,001]$ quando comparado à derivação $F Z$. Isso 
também foi encontrado durante a tarefa, com potência

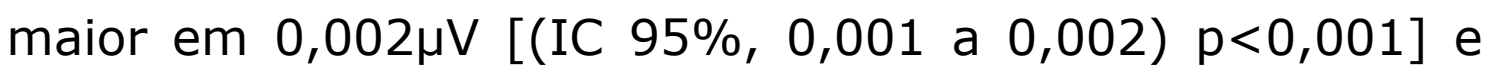
após a tarefa com potência maior em 0,002 $\mathrm{VV}$ [(IC 95\%, $0,002$ a 0,002) $p<0,001]$ na derivação F3 ao comparar a FZ.

A derivação F3 também apresentou potência maior em

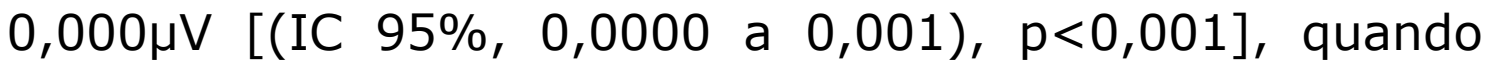
comparado à F4, antes da tarefa. No entanto, durante a

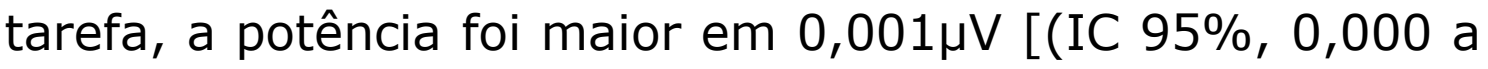
$0,001) p<0,001]$ na derivação F4. Por outro lado, após a tarefa, foi retomado o padrão de ativação inicial, com uma

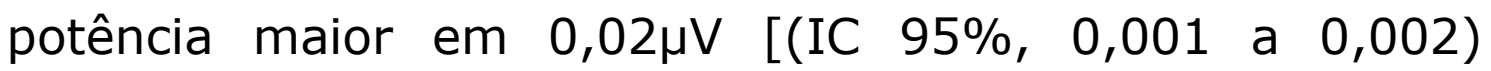
$p<0,001$ ] na derivação F3 quando comparado a F4. Em contrapartida, a derivação F4 teve uma potência maior em $0,001 \mu \mathrm{V}$ [(IC 95\%, 0,001 a 0,001) p<0,001], antes da

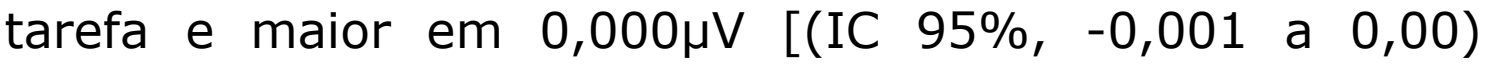
$p<0,001]$ após a tarefa, quando comparado à $F Z$.

Diferença significante também foi identificada no grupo FNP antes $\left[F(2,734)=235,803 ; p<0,001 ; \eta^{2} p=0,391\right.$; poder $=1,000]$, durante $[F(2,734)=105,075 ; \quad p<0,001$; $\eta^{2} \mathrm{p}=0,223 ; \quad$ poder $\left.=1,000\right]$ e após a tarefa $\left[F(2,734)=176,992 ; p<0,001 ; \eta^{2} p=0,325 ;\right.$ poder $\left.=1,000\right]$. O teste de post hoc mostrou que antes da realização da tarefa a derivação $\mathrm{F} 3$ teve uma potência maior em $0,002 \mu \mathrm{V}$ [(IC 95\%, 0,002 a 0,003) p<0,001] quando comparado a

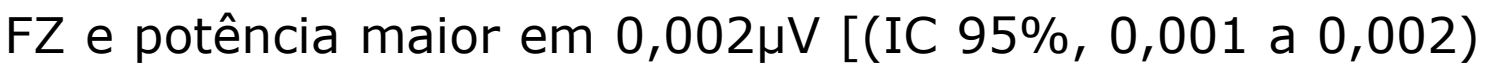
$\mathrm{p}<0,001]$ quando comparado a F4. 


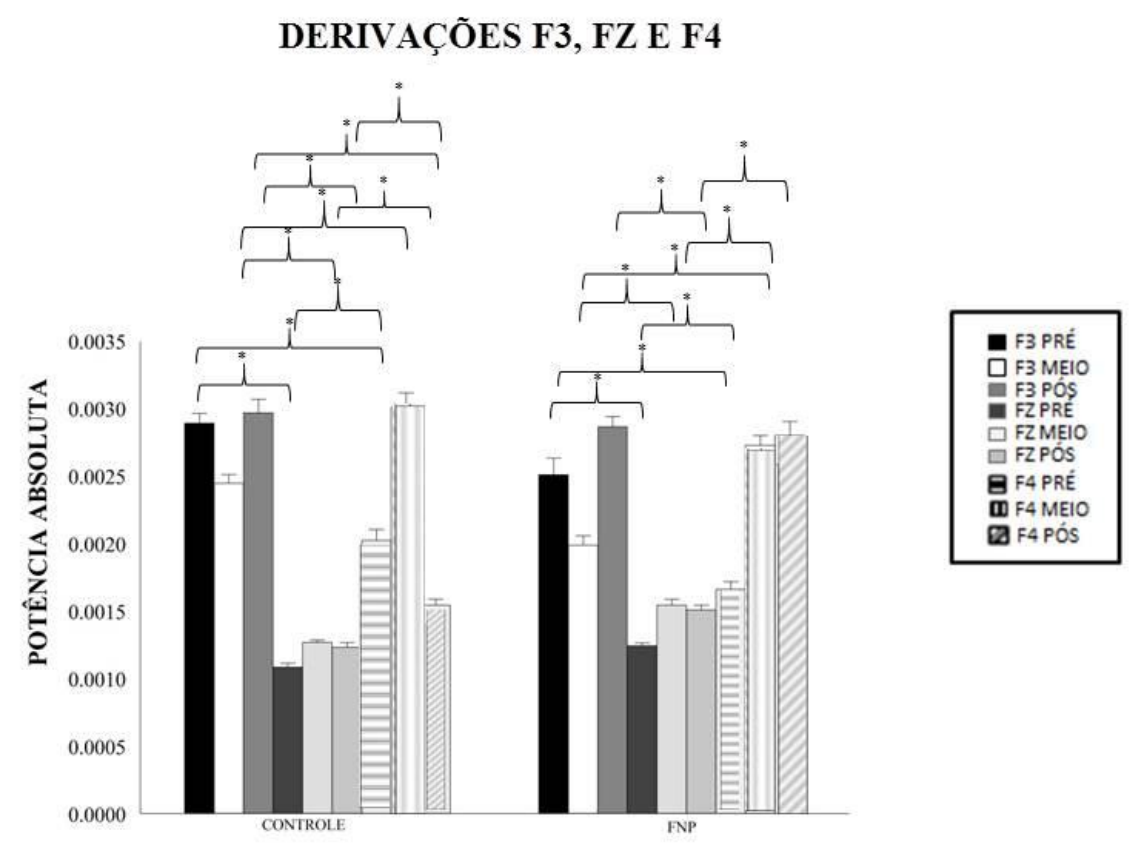

Figura 2: Média e erro padrão com diferença entre as condições controle e FNP. *Diferença significante

Ao comparar com $\mathrm{FZ}$ a potência foi maior em $0,001 \mu \mathrm{V}$ [(IC 95\%, 0,000 a 0,001) p<0,001] na derivação F4. Durante a tarefa, a derivação F3 teve uma potência maior em $0,001 \mu \mathrm{V}$ [(IC 95\%, 0,000 a 0,001) $\mathrm{p}<0,001]$ quando comparado a FZ. Por outro lado, F4 teve uma potência

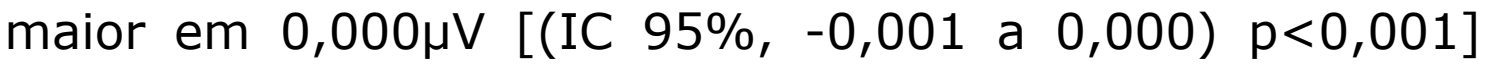

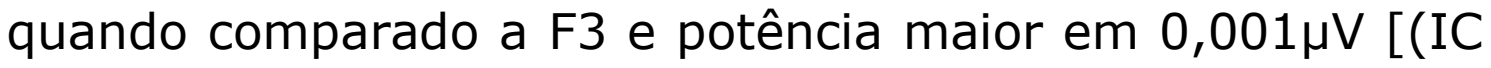
95\%, 0,001 a 0,001) $\mathrm{p}<0,001]$ quando comparado a $\mathrm{FZ}$. Após a tarefa, a derivação F3 teve uma potência maior em

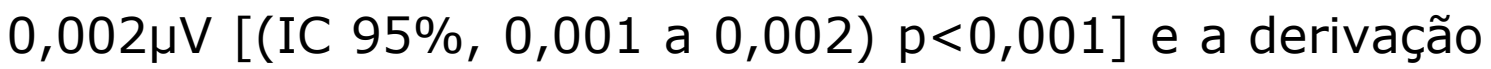
F4 teve uma potência maior em 0,002 $\mu \mathrm{V}$ [(IC 95\%, 0,001 a $0,002) \mathrm{p}<0,001]$ quando comparado a FZ. 
A análise do fator momento para a derivação F3 (Figura 3) mostrou diferença significante para o grupo controle $\left[F(2,734)=44.860 ; p<0,001 ; \eta^{2} p=0,109 ;\right.$ poder $=$ $1,000]$ e FNP $\left[F(2,734)=81,685 ; p<0,001 ; \eta^{2} p=0,182 ;\right.$ poder $=1,000]$. O teste de post-hoc mostrou um aumento

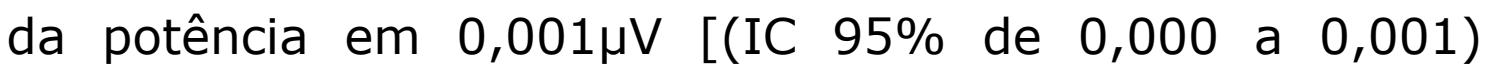
$p<0,001]$ depois da tarefa, quando comparado aos demais momentos. Por outro lado, no grupo FNP a potência foi

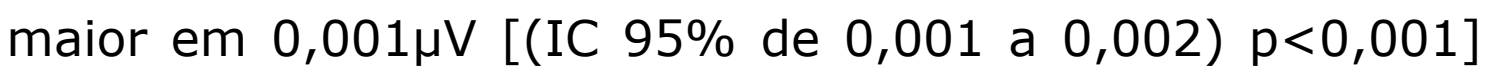
no momento antes, quando comparado ao momento durante a tarefa. No entanto, após a realização completa da

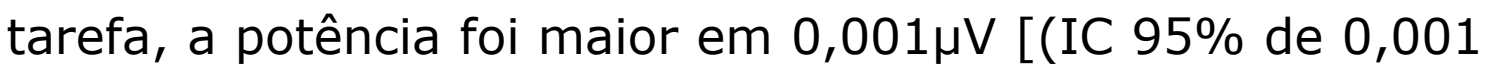
a -0,001) $\mathrm{p}<0,001]$, quando comparado ao momento durante.

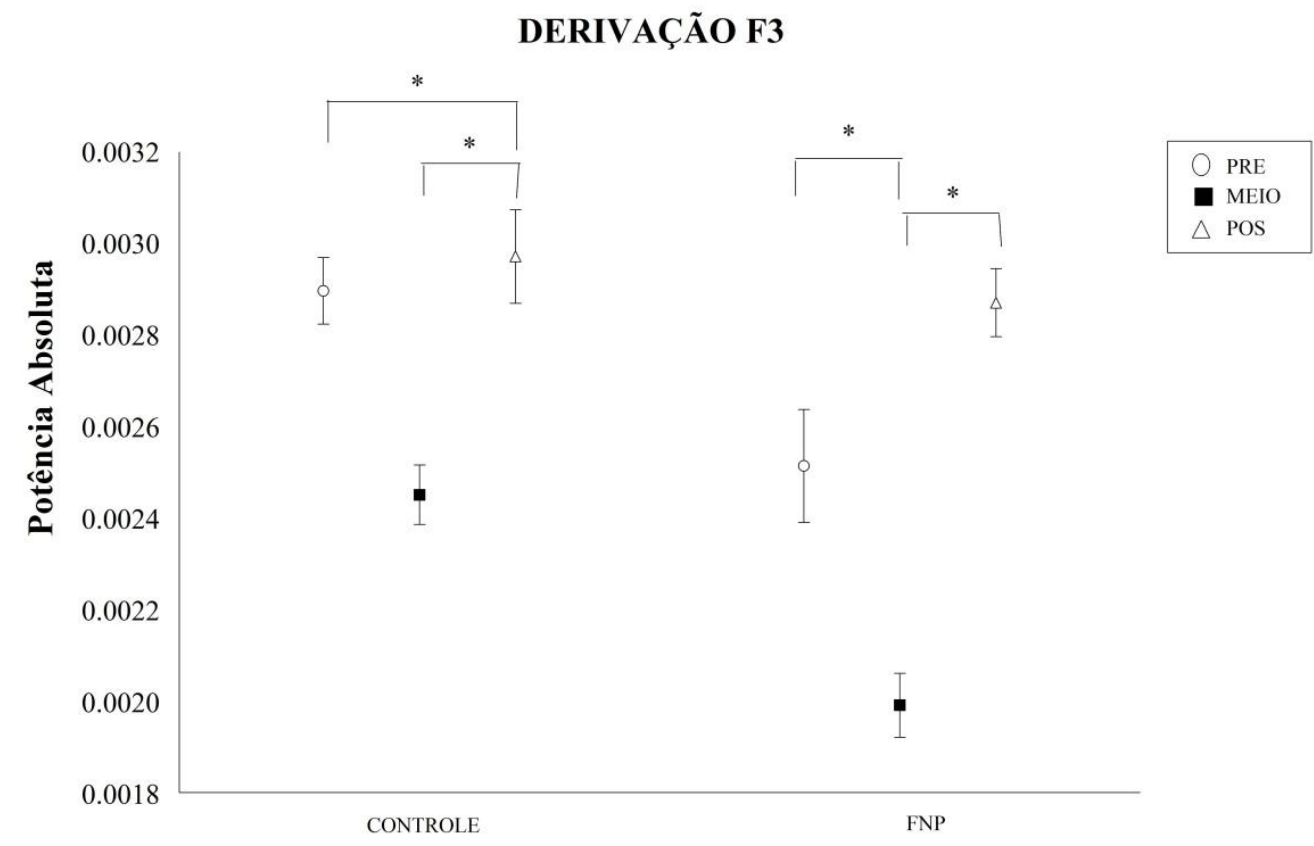

Figura 3: Média e erro padrão com diferença entre as condições controle e FNP. *Diferença significante 
Na derivação FZ (Figura 4) foi verificada diferença significativa para os grupos controle $[F(2,734)=28,113$; $\mathrm{p}<0,001 ; \quad \eta^{2} \mathrm{p}=0,071 ; \quad$ poder $\left.=1,000\right]$ e FNP $\left[F(2,734)=56,730 ; p<0,001 ; \eta^{2} p=0,134 ;\right.$ poder $\left.=1,000\right] .0$ teste post-hoc demonstrou uma potência maior em

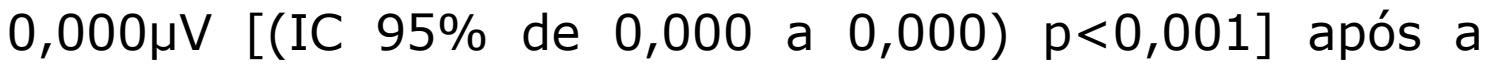
tarefa, quando comparado ao momento antes da realização da tarefa. Quando comparado o momento após ao momento durante, o aumento da potência foi de $0,000 \mu \mathrm{V}$ [(IC 95\% de - 0,005 a 0,000) p<0,001] no momento durante. No grupo FNP foi observado uma potência maior em $0,000 \mu \mathrm{V}$ [(IC 95\% de $-0,001$ a 0,001$) \quad p<0,001$ ] durante, quando comparado ao momento antes da tarefa. Da mesma forma quando comparado ao momento antes da

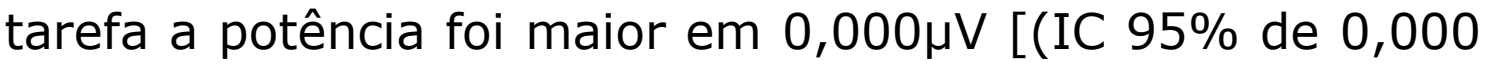
a 0,000) $p<0,001$ ] depois da realização da mesma.

Diferença significativa também foi encontrada na derivação F4 (Figura 5) para 0 grupo controle $\left[F(2,734)=104,430 ; p<0,001 ; \eta^{2} p=0,212 ;\right.$ poder $\left.=1,000\right]$ e FNP $\quad\left[F(2,734)=98,839 ; \quad p<0,001 ; \quad \eta^{2} p=0,212\right.$; poder $=1,000]$. No teste post-hoc a ativação foi maior

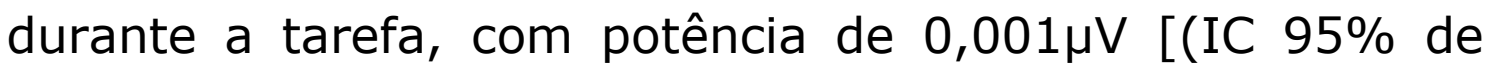
$0,001$ a 0,001) $p<0,001]$ quando comparado com o momento antes da realização da tarefa. Do mesmo modo, ao comparar com o momento após a tarefa, a potência também foi maior em $0,002 \mu \mathrm{V}$ [(IC $95 \%$ de 0,0001 a $0,002) p<0,001]$, durante a tarefa. 


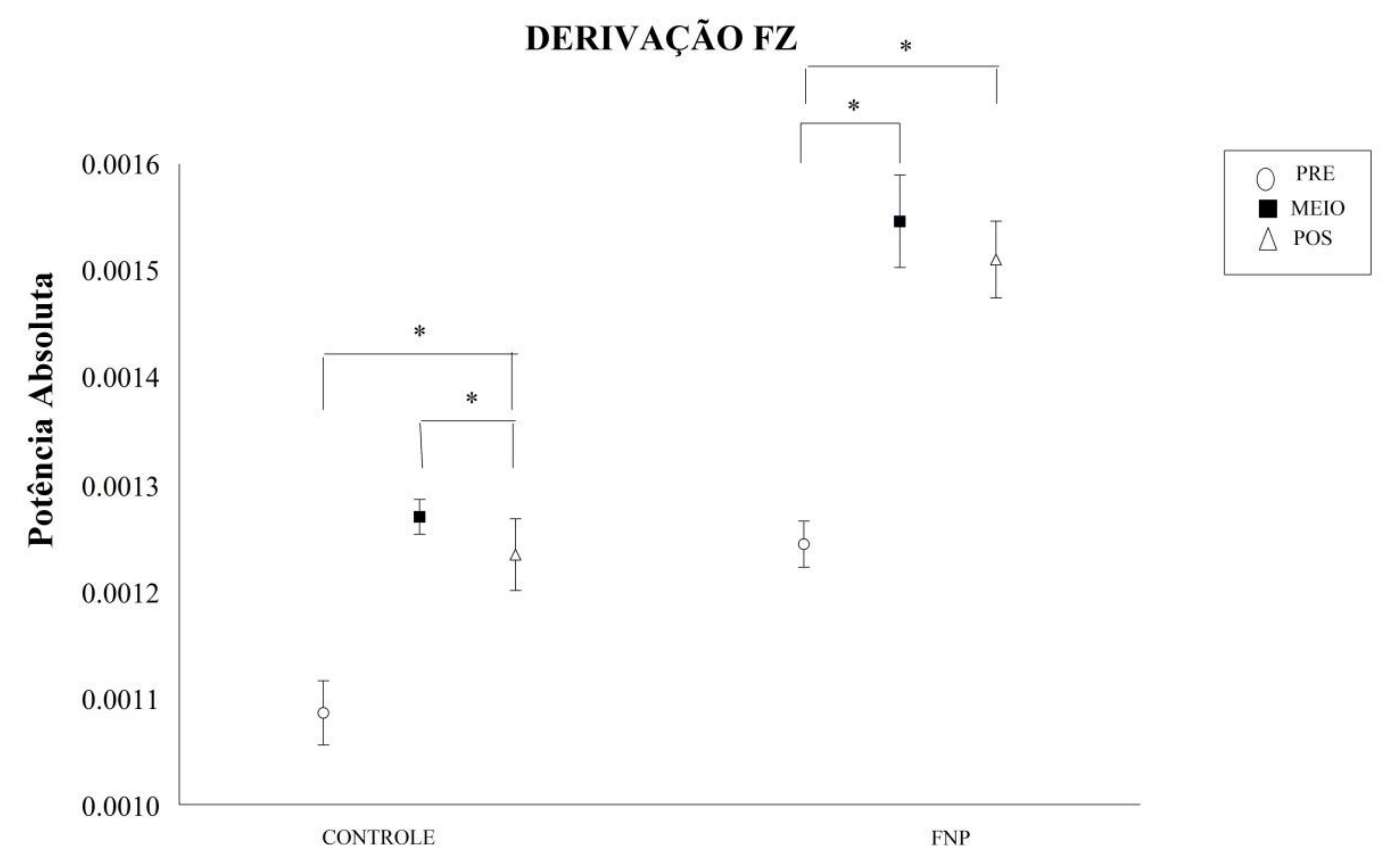

Figura 4: Média e erro padrão com diferença entre as condições controle e FNP. *Diferença significante

Ao comparar diretamente o momento antes e após a

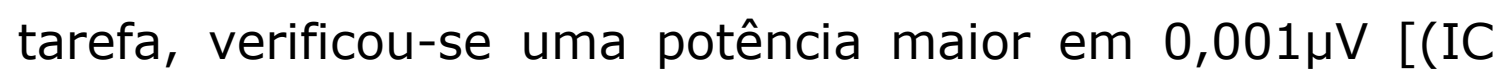
$95 \%$ de 0,000 a 0,001) $p<0,001]$ antes da tarefa. No grupo FNP foi observado maior ativação durante $0,001 \mu \mathrm{V}$ [(IC 95\% de 0,001 a 0,001) p<0,001] quando comparado com o momento antes da tarefa. Do mesmo modo, a potência

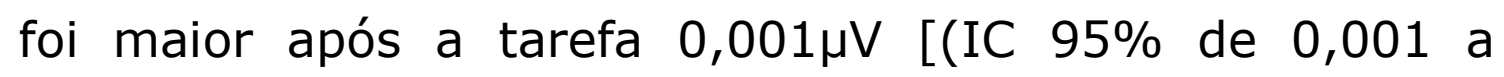
$0,002) \mathrm{p}<0,001]$ quando comparado com o momento antes e maior em 0,000 $\mathrm{V}$ [(IC 95\% de $-0,001$ a 0,000) $\mathrm{p}<0,001]$, ao comparar com o momento durante a tarefa. 


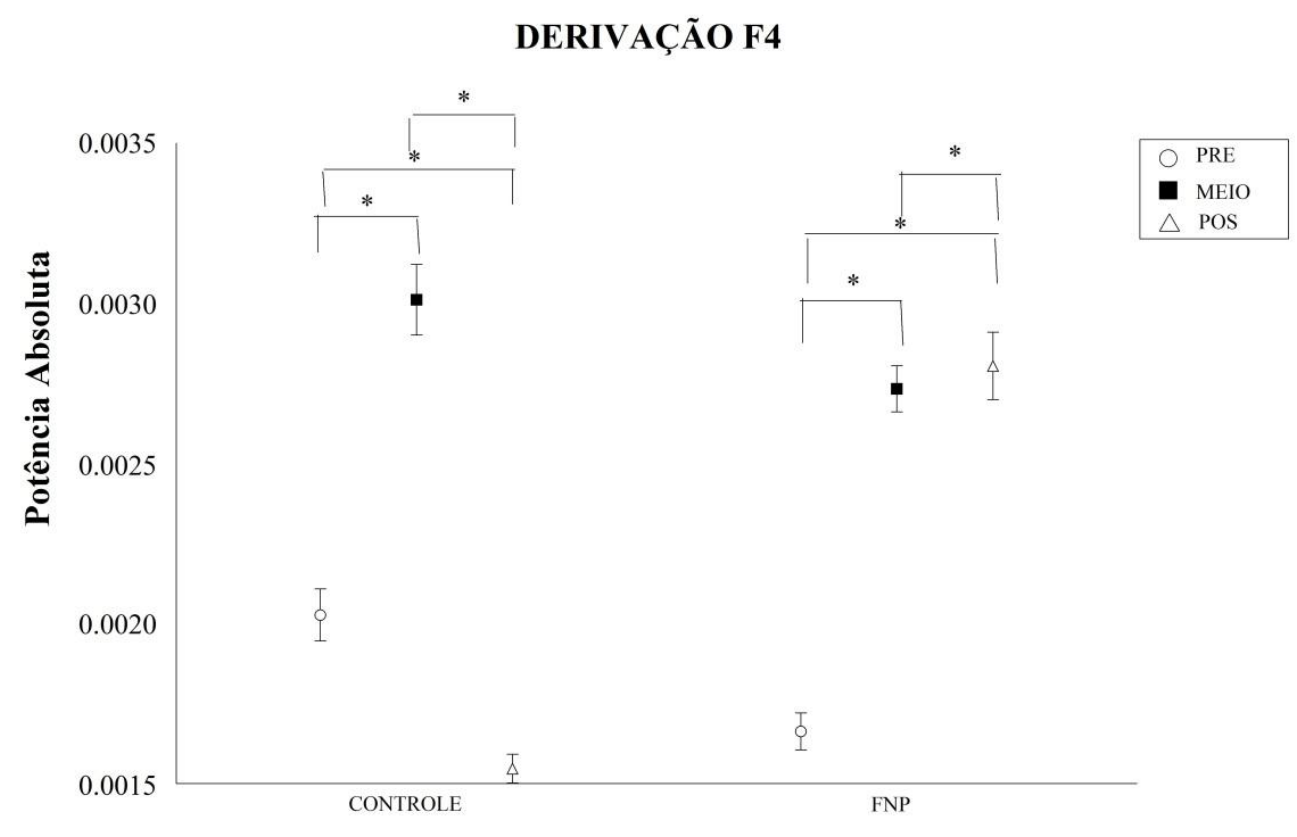

Figura 5: Média e erro padrão com diferença entre as condições controle e FNP. *Diferença significante

\section{DISCUSSÃO}

O presente estudo visa esclarecer a dinâmica eletrofisiológica cortical da potência absoluta na banda gama no córtex pré-frontal após a tarefa de movimento em diagonal do membro superior direito. A banda gama corresponde a uma frequência entre $30 \mathrm{~Hz}$ a $100 \mathrm{~Hz}$ e está envolvida no processo cognitivo, memória, e fatores de integração espacial/temporal e proprioceptivos ${ }^{24,36,37}$. Em consonância, a atividade gama tem sido relacionada com o processo sensório-motor durante o desempenho de tarefas 
que envolvem a discriminação visual e preparação motora $22,27,38,39$.

O córtex pré-frontal desempenha um papel essencial na formação de metas e objetivos, e no planejamento de estratégias de ação necessárias para a consecução destes objetivos, selecionando as habilidades cognitivas requeridas para a implementação dos planos, e coordenando as mesmas para aplicá-las na ordem correta ${ }^{40-42}$. As derivações F3, FZ e F4 representam o córtex pré-motor, funcionalmente responsáveis pela seleção de movimentos, preparação e controle voluntário da ação $22,43,44$.

Avaliando-se o grupo FNP, da mesma forma que no grupo controle, oobservou-se que antes e após a realização da tarefa houve um maior aumento da potência absoluta no córtex pré-frontal dorsolateral esquerdo (F3) quando comparado às demais áreas e uma maior potência no córtex pré-frontal dorsolateral direito (F4) durante a tarefa. $O$ aumento da potência na derivação $\mathrm{F} 3$ em relação às demais derivações antes da realização da tarefa pode estar relacionado com o envolvimento do córtex pré-frontal dorsolateral esquerdo durante o planejamento e preparação de uma ação motora envolvendo o membro superior direito ${ }^{45,46}$. Em relação a maior ativação na derivação F4 durante a realização da tarefa, os resultados obtidos podem ser confirmados por estudos que apontam uma ativação no córtex pré-frontal direito em tarefas de evocação ${ }^{47}$ ao passo que o córtex pré-frontal esquerdo mostra-se mais ativado em tarefas de reconhecimento ${ }^{27,48}$. 
O aumento de potência observado na derivação F3 antes mesmo da realização da tarefa pode ser explicada por achados na literatura ao afirmarem que quando o sujeito recebe informação sobre o que deverá aprender (processamento cognitivo) a dinâmica eletrofisiológica cortical sofre aumento de potência por exigir ativação cortical que gera atenção para aquisição cognitiva da tarefa motora; e que a prática deliberada da tarefa (comportamento motor) exige um estado eletrofisiológico cortical menos ativado em relação às tarefas puramente cognitivas, corroborando os resultados de pequena ativação no momento durante a realização da tarefa, no entanto ainda maior do que o estado antes da realização da tarefa, por exigir ativação cortical que gera atenção para aquisição motora e evocação de memória cognitiva e motora, para identificação visual e cognitiva para a execução da tarefa 27,49 , achados esses que refutam os resultados do presente estudo o qual mostrou uma maior ativação antes da realização da tarefa em comparação aos demais momentos. Por outro lado o aumento da potência no momento após a tarefa pode ocorrer devido à aquisição da informação motora e processo de consolidação durante o comportamento motor refletindo o aumento de potência na banda em questão 22,50 .

O aumento na potência absoluta da banda gama, na derivação $F Z$, durante a tarefa pode estar relacionada ao envolvimento do córtex pré-frontal no processamento visuo-espacial $^{51,52}$ percepção visual e processos de 
identificação e atenção aos estímulos gerando maior repercussão em comparação às demais áreas. O mesmo também foi encontrado após a realização completa da tarefa, onde a potência também aumentou mostrando que quanto mais o sujeito realiza a tarefa, maior a atividade cortical. Isso pode ocorrer porque a tarefa demanda muita atenção e envolve a presença de estímulos para a sua iniciação.

Do mesmo modo, o maior aumento da potência absoluta na banda gama na derivação F4 no momento após a tarefa, como visto anteriormente, pode estar relacionado a tarefas de evocação que, segundo a literatura, ativa o córtex pré-frontal direito. De modo geral, o aumento da potência absoluta na banda gama no córtex pré-frontal se deve a relação da mesma aos processos sensorial e cognitivo do controle motor, ao seu envolvimento em atenção, planejamento, seleção de movimentos, elaboração e controle voluntário da ação ${ }^{44}$, e ao papel do córtex préfrontal na organização do comportamento e nas ações cognitivas $^{42,53,54}$.

\section{CONCLUSÃO}

No presente estudo analisaram-se quais as repercussões eletrofisiológicas dos movimentos em diagonal por meio da análise da potência absoluta na banda gama no córtex pré-frontal e diante disso concluiu-se que embora se tenha observado um aumento da potência 
absoluta na banda gama, à medida que o sujeito realizava a tarefa, tanto no grupo controle quanto no grupo FNP, este produziu maior ativação cortical mostrando-se eficiente em modular a atividade neural. Desse modo, tais achados mostraram que esse método de tratamento é eficiente tanto em nível muscular quanto em nível cortical, o que justifica sua utilização na prática clínica. Os resultados, por sua vez, corroboram com a hipótese de que a realização da tarefa por meio do movimento em diagonal promove 0 desencadeamento do potencial neuromuscular, provocando alterações específicas na atividade cortical do cérebro, resultando em aumento da ativação cortical ao longo da tarefa. No entanto, o aumento da potência absoluta na banda gama em ambos os grupos (controle e FNP) sugere que o incremento da potência não esteja relacionado isoladamente à tarefa de diagonal do membro superior, o que leva a necessidade da realização de estudos futuros a fim de complementar os achados do presente artigo.

\section{REREFÊNCIAS}

1.Westwater-Wood S, Adams N, Kerry R. The use of Proprioceptive Neuromuscular Facilitation in physiotherapy practice. Phys Therp Rev 2010;15:23-8. https://doi.org/10.1179/174328810X12647087218677 2.Alencar RF, Cordeiro TGF, Anjos PGS, Cavalcanti PL. Facilitação Neuromuscular Proprioceptiva em tatame na reaquisição de funções na lesão medular. Rev Neurocienc 2011;19:512-8.

3. Hindle KB, Whitcomb TJ, Briggs WO, Hong J. Proprioceptive Neuromuscular Facilitation (PNF): Its Mechanisms and Effects on Range of Motion and Muscular Function. J Hum Kinet 2012;31:10513. https://doi.org/10.2478/v10078-012-0011-y

4.Lee SW, Lee JH. Effects of Proprioceptive Neuromuscular Facilitation Stretching and Kinesiology Taping on Pelvic Compensation During Double-Knee Extension. J Hum Kinet 2015;49:55-64. https://doi.org/10.1515/hukin-2015-0108 
5.Gontijo LB, Pereira PD, Neves CD, Santos AP, Machado Dde $\mathrm{C}$, Bastos VH. Evaluation of Strength and Irradiated Movement Pattern Resulting from Trunk Motions of the Proprioceptive Neuromuscular Facilitation. Rehabil Res Pract 2012;2012:281937. https://doi.org/10.1155/2012/281937

6. Kim CH, Kim YN. Effects of Proprioceptive Neuromuscular Facilitation and Treadmill Training on the Balance and Walking Ability of Stroke Patients. J Kor Phys Ther 2018:30:79-83. https://doi.org/10.18857/jkpt.2018.30.3.79

7.Lazarou L, Kofotolis N, Pafis G, Kellis E. Effects of two proprioceptive training programs on ankle range of motion, pain, functional and balance performance in individuals with ankle sprain. J Back and Musculoskelet Rehabil 2018;31:437-46. https://doi.org/10.3233/BMR-170836

8.Kim Y, Kim E, Gong W. The effects of trunk stability exercise using PNF on the functional reach test and muscle activities of stroke patients. J Phys Ther Sci 2011;23:699-702. https://doi.org/10.1589/jpts.23.699

9.Yoo B, Park H, Heo K, Lee J, Lee J, Oh T, et al. The Effects of Abdominal Hollowing in Lower-limb PNF Pattern Training on the Activation of Contralateral Muscles. J Phys Ther Sci 2013;25:1335-8. https://doi.org/10.1589/jpts.25.1335

10.Ferreira MG, Bertor WRR, Carvalho AR, Bertolini GRF. Effects of static, ballistic, and proprioceptive neuromuscular facilitation stretching on vertical jump variables. Sci Med 2015;25:ID21443. http://dx.doi.org/10.15448/1980-6108.2015.4.21443

11.Seo KC, Kim HA. The effects of ramp gait exercise with PNF on stroke patients dynamic balance. J Phys Ther Sci 2015; 27:1747-9. https://doi.org/10.1589/jpts.27.1747

12.Aman JE, Elangovan N, Yeh I-L, Konczak J. The effectiveness of proprioceptive training for improving motor function: a systematic review. Front Human Neurosci 2015;8:1075. https://doi.org/10.3389/fnhum.2014.01075

13.Vogt $T$, Kato $K$, Fluthmann $N$, Bloch $O$, Nakata $H$, Kanosue $K$. Performance control in one consecutive motor task sequence Approaching central neuronal motor behaviour preceding isometric contraction onsets and relaxation offsets at lower distinct torques. J Musculoskelet Neuronal Interact 2018;18:1-8.

14.Oswal A, Litvak V, Brücke C, Huebl J, Schneider GH, Kühn AA, et al. Cognitive Factors Modulate Activity within the Human Subthalamic Nucleus during Voluntary Movement in Parkinson's Disease. J Neurosci 2013;33:15815-26.

https://doi.org/10.1523/JNEUROSCI.1790-13.2013

15.Owolabi L, Sale S, Owolabi S. Clinico-Electroencephalography Pattern and Determinant of 2-year Seizure Control in Patients with Complex Partial Seizure Disorder in Kano, Northwestern Nigeria. Ann Med Health Sci Res 2014:4:186-91. https://doi.org/10.4103/2141$\underline{9248.129030}$ 
16. Reinke $\mathrm{L}$, van der Hoeven $\mathrm{JH}$, van Putten MJ, Dieperink W, Tulleken JE. Intensive care unit depth of sleep: proof of concept of a simple electroencephalography index in the non-sedated. Crit Care 2014;18:R66. https://doi.org/10.1186/cc13823

17. Carvalho MR, Velasques BB, Cagy M, Marques JB, Teixeira S, Nardi $A E$, et al. Electroencephalographic findings in panic disorder. Trends Psychiatry Psychother 2013; 35:238-51.

https://doi.org/10.1590/2237-6089-2013-0012

18.Cunha M, Machado S, Miana LC, Machado D, Bastos VH, et al. Effects of a cognitive modulator in the theta and alpha asymmetry during a typewriting task. Arq Neuropsiquiatr 2009;67:214-8. http://dx.doi.org/10.1590/S0004-282X2009000200008

19. Machado DCD, Santos RPMS, Silva AP, Reis-Filho SBS, Aves GVS, Cagy $\mathrm{M}$, et al. Electroencephalographic analysis in left hemiparesis: a case study. Rev Bras Neurol 2013;49:129-6.

20.Mosanezhad JE, Nazari MA. Effectiveness of EEG-Biofeedback on Attentiveness, Working Memory and Quantitative Electroencephalography on Reading Disorder. Iran J Psychiatry Behav Sci 2013;7:35-43.

21. Nasseroleslami B, Lakany $H$, Conway BA. EEG signatures of arm isometric exertions in preparation, planning and execution. Neuroimage 2015;90:1-14.

http://dx.doi.org/10.1016/j.neuroimage.2013.12.011

22. Machado D, França JHS, Teixeira S, Basto VHV, Santos RPM, Cagy $M$, et al. Gamma absolute power reveals activation of motor áreas after hand immobilization. MedicalExpress (Sao Paulo, online) 2016;3:M160504.http://dx.doi.org/10.5935/MedicalExpress.2016.05. $\underline{04}$

23.Bonini-Rocha AC, Timm MI, Chiaramonte M, Zaro M, Rasia-Filho $A A$, Wolff $D$, et al. Methodology to observation and quantification of EEG signs related to cognitive evidences of motor learning. Ciências \& Cognição 2008;13:27-50.

24.van Es MWL, Schoffelen JM. Stimulus-induced gamma power predicts the amplitude of the subsequente visual evoked response. NeuroImage 2019;186:703-12.

http://dx.doi.org/10.1016/j.neuroimage.2018.11.029

25. Teixeira S, Velasques B, Machado S, Cunha M, Domingues $\mathrm{CA}$, Budde $\mathrm{H}$, et al. Gamma-band oscillations in fronto-central areas during performance of a sensorimotor integration task: A qEEG coherence study. Neurosci Lett 2010;483:114-7. http://dx.doi.org/10.1016/j.neulet.2010.07.073

26.Grossmann T, Johnson MH, Lloyd-Fox S, Blasi A, Deligianni $F$, Elwell $C$, et al. Early cortical specialization for face-to-face communication in human infants. Proc Biol Sci 2008;275:2803-11. http://dx.doi.org/10.1098/rspb.2008.0986

27.Silva AAS, Trindade-Filho EM. Diferenças no processamento cerebral, através do ritmo gama, durante o pensamento divergente. Rev Neurocienc 2015;23:589-94.

http://dx.doi.org/10.4181/RNC.2015.23.04.968.06p 
28. Oldfield RC. The assessment and analysis of handedness: the Edinburg inventory. Neuropsychologia 1971;9:97-113.

29. Hallal P, Andersen LB, Bull FC, Guthold R, Haskell W, Ekelund U, et al. Global physical activity levels: surveillance progress, pitfalls, and prospects. Lancet 2012;380:247-57.

http://dx.doi.org/10.1016/S0140-6736(12)60646-1

30.World Health Organization. Physical Status: the use of interpretation of anthropometry. Report of a Who Expert Committee (endereço na internet). Geneva: WHO, 1995. (acessado em 2018). Disponível https://www.who.int/childgrowth/publications/physical_status/en/

31.Delorme A, Makeig S. EEGLAB: an open source toolbox for analysis of single-trial EEG dynamics including independent component analysis. J Neurosci Methods 2004;134:9-21.

32.Jung TP, Makeig S, Humphries C, Lee TW, McKeown MJ, Iragui V, et al. Removing electroencephalographic artifacts by blind source separation. Psychophysiology 2000;37:163-78.

33.Delorme A, Palmer J, Onton J, Oostenveld R, Makeig S. Independent EEG Sources are Dipolar. PLoS ONE 2012;7:e30135. http://dx.doi.org/10.1371/journal.pone.0030135

34. Hopkins W, Marshall S, Batterham A, Hanin J. Progressive statistics for studies in sports medicine and exercise science. Med Sci Sports Exerc 2009;41:3-13.

http://dx.doi.org/10.1249/MSS.0b013e31818cb278

35.Fayers M, Machin D. Sample size: how many patients are necessary? Br J Cancer 1995;72:1-9.

36. Herrmann CS, Fründ I, Lens D. Human gamma-band activity: a review of cognitive and behavioral 86 correlates and network models. Neurosci Biobehav Rev 2010;34:981-92.

http://dx.doi.org/10.1016/j.neubiorev.2009.09.001

37. Howard MW, Rizzuto DS, Caplan JB, Madsen JR, Lisman J, Aschenbrenner-Scheibe $\mathrm{R}$, et al. Gamma oscillations correlate with working memory load in humans. Cereb Cortex 2003;13:1369-75. https://doi.org/10.1093/cercor/bhg084

38.Sinai CW, Bowers CW, Crainiceanu CM, Boatman D, Gordon $\mathrm{B}$, Lesser RP, et al. Electrocorticographic high gamma activity versus electrical cortical stimulation mapping of naming. Brain 2005;128:1556-70. https://doi.org/10.1093/brain/awh491

39.Slobounov S, Hallet M, Stanhope S, Shibasaki H. Role of cerebral cortex in human postural control: an EEG study. Clin Neurophysiol 2005;116:315-23. https://doi.org/10.1016/j.clinph.2004.09.007

40.Fuster JM. The prefrontal cortex. 4th ed. London: Academic Press, 2008, 460p.

41.Mourão Junior CA, Melo LBR. Integration of three concepts: executive function, working memory and learning. Psicologia: Teoria e Pesquisa 2012;27:309-14. https://doi.org/10.1590/S0102$\underline{37722011000300006}$ 
42. Ackerman CM, Courtney SM. Spatial relations and spatial locations are dissociated within pré-frontal and parietal córtex. J Neurophysiol 2012;108:2419-29. https://doi.org/10.1152/jn.01024.2011

43. Machado S, Cunha M, Portella CE, Silva JG, Velasques B, Bastos $\mathrm{VH}$, et al. Integration of cortical areas during performance of a catching ball task. Neurosci Left 2008;446:7-10. https://doi.org/10.1016/j.neulet.2008.09.036

44. Velasques S, Machado S, Portella CE, Silva JG, Basile LF, Cagy M, et al. Electrophysiological analysis of a sensorimotor integration task. Neurosci Lett 2007;426:155-9.

https://doi.org/10.1016/j. neulet.2007.08.061

45.Szurhaj W, Derambure P, Labyt E, Cassim F, Bourriez JL, Isnard J, et al. Basic mechanisms of central rhythms reactivity to preparation and execution of a voluntary movement: a stereoelectroencephalographic study. Clin Neurophysiol 2003;114:107-19. https://doi.org/10.1016/S1388-2457(02)00333-4

46. Barbey AK, Koenigs M, Grafman J. Dorsolateral Prefrontal Contributions to Human Working Memory. Cortex 2013;49:1195-205. https://doi.org/10.1016/j.cortex.2012.05.022

47.Nyberg L, Cabeza R, Tulving E. PET studies of encoding and retrieval: the HERA model. Psychonomic Bull Rev 1995;3:135-48. https://doi.org/10.3758/BF03212412

48. Thach WT. On the specific role of the cerebellum in motor learning and cognition: clues from PET activation and lesion studies in man. Behav Brain Sci 1996;19:411-31.

https://doi.org/10.1017/S0140525X00081504

49.Bressler SL. The gamma wave: a cortical information carrier? Trends Neurosci 1990;13:161-2. https://doi.org/10.1016/01662236(90)90039-D

50.Bonini-Rocha AC, Timm MI, Chiaramonte MS, Zaro MA. Metodologia para observação e quantificação de sinais de EEG relativos a evidências cognitivas de aprendizagem motora. Ciências \& Cognição 2008;13:27-50.

51.Medendorp WP, Goltz HC, Vilis T, Crawford JD. Gaze-centered updating of visual space in human parietal cortex. J Neurosci 2003;23:6209-14. https://doi.org/10.1523/JNEUROSCI.23-1506209.2003

52.Avillac M, Deneve S, Olivier E, Pouget A, Duhamel JR. Reference frames for representing visual and tactile locations in parietal cortex. Nat Neurosci 2005;8:941-9.

53.Mourão-Junior CA, Melo LBR. Integração de Três Conceitos: Função Executiva, Memória de Trabalho e Aprendizado. Psic: Teor e Pesq 2011;27:309-14. http://dx.doi.org/10.1590/S010237722011000300006

54.Moreno MA, Silva E, Zuttin RS, Gonçalves M. Efeito de um programa de treinamento de facilitação neuromuscular proprioceptiva sobre a mobilidade torácica. Fisioter Pesq 2009;16:161-5. http://dx.doi.org/10.1590/S1809-29502009000200012 